\title{
PHYSICAL SIMULATION OF LONG DISTANCE AND DIRECTIONAL WIRELESS CHANNELS
}

\author{
Christopher Meagher, Randall Olsen, Christopher Cirullo, Christina de Jesus, and Robert C. Ferro \\ SPAWAR Systems Center Pacific (SSC Pacific) \\ San Diego, CA
}

\begin{abstract}
In order to accurately test directional or multiplexed systems in a lab environment, a testbed should simulate both directional wireless channels and propagation delay. Using one or more RF lenses, one can test a radio system that uses switched or adaptively steerable antennas: one side is switched by an "environment" controller, the other by the the discovery and tracking loop of the radio system. True path delay testing can be done by using analog $R F$ to-optical converters and miles of low-cost, low-loss fiber optics. Apart from verifying latency, this test can also determine if there are issues with ACK or other timeouts: information unavailable by simply attenuating a link to simulate distance.
\end{abstract}

\section{INTRODUCTION}

There is great interest in leveraging commercial off-theshelf (COTS) wireless technology to bolster military communication capabilities. However, these COTS radios are typically for home, school, or office use - all which are short distance and can tolerate moderate latency and reliability. On the other hand, military communications typically require long distance, low latency, and high reliability links.

Use of directional antennas and frequency or spatial multiplexing are some of the approaches used to bridge this gap between COTS technologies and military use. Directional antennas offer increased range, but they have to be steered to accommodate military mobility. For frequency and spatial multiplexing, several low-cost WiFi and emerging WiMAX radios can be tied to different directional antennas or sectors for integration into one communications system. In this paper, we discuss these architectures, the need for physical simulation of both a directional and long distance wireless channel, and describe the current implementation at the Space and Naval Warfare (SPAWAR) Systems Center Pacific (SSC Pacific).

\section{WIRELESS ARCHITECTURES}

Apart from increased security, the most important requirement for wireless links to be used in military applications is the ability to work at long distances. Since most COTS radios are limited in power to hundreds of milliwatts, long distance links must be achieved by increasing gain in either additional amplifiers or in the antennas.

If it is possible to place an omni-directional antenna on top of the platform, high gain power amplifiers can be used to transmit long distances. This architecture has been the solution for most line-of-sight (LOS) military communications systems. Low noise amplifiers (LNAs) could also be added to the RF front end of a system, but the benefits are eventually limited to the noise figure of the COTS radio that is being used. Furthermore, such added LNAs would require additional high order filters as protection from the harsh EMI environment on many military platforms (e.g. RADARs aboard ships and aircraft).

There are many instances were a single, omni-directional antenna is undesirable or unfeasible. Costly power amplifiers, a crowded topside, and the desire to reduce probably of detection/jamming all tend to favor a second type of architecture that uses high gain directional antennas and multiple radios. Common examples of directional antennas are parabolic dishes or grids, periodic wire antennas, and fixed or phased arrays. The last of these types has been uncommon in communications but is of significance in this paper as described in the "Addressing the Directional Channel" section.

The notion of using multiple radios for a single node is also relatively novel and runs counter to the paradigm on most military platforms, even the largest ones. However, contrary to high cost, stovepipe military radios, COTS radios tend to be inexpensive enough to allow for a single platform to have several, including spares. Having multiple radios allows them to be placed near their corresponding directional antenna, thus removing the need 
for expensive, lossy RF cable runs. The only drawback in this second architecture is the need for software to keep track of which radios are being used [1].

\section{THE NEED FOR PHYSICAL SIMULATION}

Once the radio or architecture has been chosen, it is important to test whether it will work in the intended operational environment. At the very least, this means testing through the link budget and timing issues. For the multiple radios architecture or for one that steers one or more directional antennas, control software also needs to be iteratively developed and tested.

For both proof-of-concept and even prototyping, purely software-based simulation can be helpful. Two of the most used communications and network simulation tools are OPNET and Qualnet. Both can model radios according to their standards (e.g. 802.11a, 802.16e) and the system models can be edited to include any modifications. Furthermore, they can model some propagation and terrain effects and even allow users to define switched or steerable directional antenna patterns [2], [3].

Drawbacks to the software-only approach to simulation include high costs, limited hardware-in-the-loop options, and steep learning curves. The last point consists not only of learning how to use the software and modify radio models but also of learning the intricacies of the actual radio one wishes to model. This can be extremely difficult, especially with COTS radios that are pre-standard (e.g. draft-802.11n and pre-WiMAX) or that add to or modify standards-compliant radios.

On the other hand, using hardware in a near-operational environment is prohibitively expensive for proof-ofconcept and development testing. A middle ground between software-only simulation and operational environment testing is to test the actual radios using physical hardware to simulate the environment. Of course, such a system can be made very intricate and costly in order to simulate all aspects of the environment. However, low cost systems, as will be described below, can address two first-order questions: can the system work directionally and can it work at long distance?

\section{SIMULATING THE DIRECTIONAL CHANNEL}

The rule for having two omni-directional nodes communicate is easy: they must lie within each other's coverage area. The rule becomes more complex with directional antennas, which have to point to each other during discovery and move or change beams for tracking of mobile nodes. This is further complicated with time division multiplexing (TDM) networks, which must coordinate transmit and receive along with beam pointing. Various algorithms for directional mobile ad hoc networks (MANETs) have been discussed in the literature [4], [5], [6]. However, few if any have been tested with real radios or in any physical environment.

RF lenses provide a compact way to simulate propagation of directional beams. Rather than radiating into the freespace of an indoor or outdoor antenna range, two lenses can be placed back-to-back to simulate one-dimensional transmit and receive beam steering. The lenses generate a phase slope, $\varphi_{n}$, that - if feeding a linear array antenna would create a beam at some angle, $\theta_{s}$ :

$$
\theta_{s}=\sin ^{-1}\left[\frac{\phi_{n} \lambda}{2 \pi n \Delta_{x}}\right]
$$

where $\lambda$ is the wavelength, $n$ is the number of elements, and $\Delta_{x}$ is the inter-element spacing [7]. By reciprocity, the same beam impinging upon a linear array antenna would generate a phase slope of $-\theta_{s}$. So, by instead connecting the two lenses together with phase-matched cables, freespace propagation can be avoided. The figure below shows simulations of two microstrip Rotman lenses in such a configuration as well as the currents for nearbroadside and $40^{\circ}$ propagation.

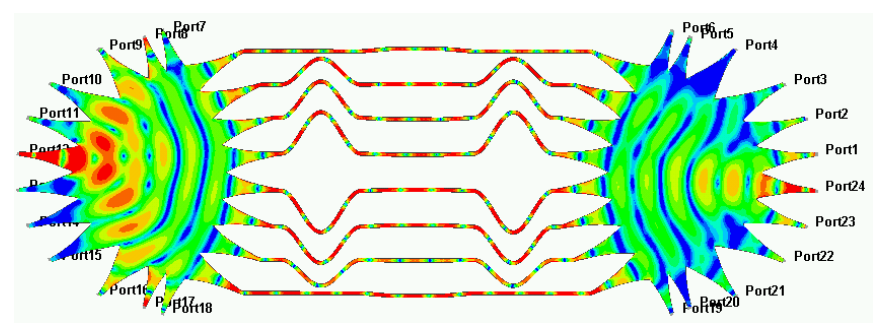

(a) Near-Broadside

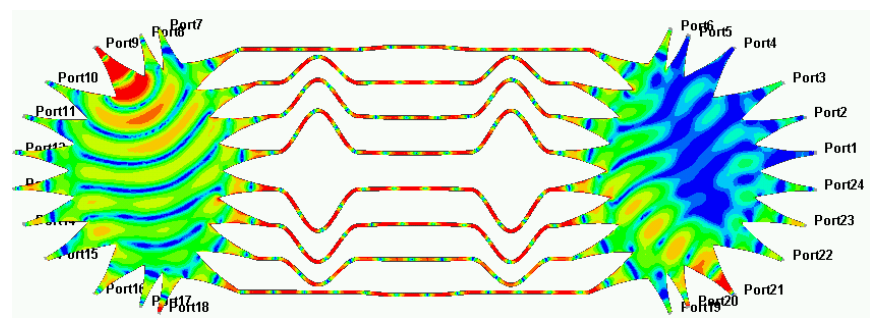

(b) $40^{\circ}$ Off Broadside

Figure 1: Back-to-Back Rotman Lenses Simulating Directional Propagation 
The above figure shows beam-forming lenses with switched beams. The number of beams and their angles are fixed. One could also generate a continuouslysteerable directional channel by adding phase shifters between the antenna ports of the two lenses above or by starting with steerable-beam RF lenses. The latter could be done with horns that slide across the feed contour of parallel plate waveguide lenses, as were originally envisioned by Rotman and Turner [8]. Such continuous variation would more accurately simulate the mechanical movement of tracking satellite dishes, if only in one dimension. Two-dimensional simulation could be done as it would with steering, by the use of two generations of RF lenses, one for each dimension. However, at this point the testbed has become expensive and overly complex. Considering that most LOS communications occur within a $10^{\circ}$ vertical region about the horizon $-20^{\circ}$ when considering rough seas or terrain - one-dimensional steering is often acceptable.

For simpler wireless systems, just a variable attenuator or two multi-pole, single throw RF switches and various fixed attenuators can be used. For example, a system might consist of one radio that can switch amongst six different, $60^{\circ} \mathrm{AZ}$ patch antennas that have fixed pointing angles. If two nodes are simulated to be collinear and their chosen antennas are pointed at each other, the switched-in attenuation is $0 \mathrm{~dB}$. If a node is off by one position, the gain of its antenna would have rolled off by $3 \mathrm{~dB}$, so $3 \mathrm{~dB}$ of attenuation is switched in. If both nodes are off by one position in the same direction, a total of $6 \mathrm{~dB}$ of loss is added, and so on.

Finally, a controller is needed to test the system in all of its many possible environments. This environment controller consists of software that varies the link attenuation to simulate non-optimal antenna pointing. In the simple variable attenuator setup described above, this could be a routine that compares the antenna switch control of the system under test (SUT) with that of the simulated remote node and switches in attenuation accordingly. When two RF lenses are placed back-to-back, the attenuation is affected by beam forming. The environment controller steers one lens to simulate the position of the remote node; then, it is up to the SUT to sweep across the beams on its $\mathrm{RF}$ lens and choose the best pointing direction.

\section{ADDRESSING THE LONG DISTANCE CHANNEL}

An additional complication to using COTS wireless technology in military applications is that the latter often requires long distance communications and robustness to fading and delay. On the other hand, commercial and home-use systems automatically assume short distances and redundant coverage and try to optimize the user's experience under those assumptions. For example, this allows some 802.11 radios to "roam" from access point to access point. And, though useful in a campus setting, the "slow scan"mode that these radios enter below a certain signal-to-noise (SNR) threshold can cause unnecessary latency in a military link [1].

Many researchers simulate increasing distance in a link by increasing the attenuation in either cabled or close proximity wireless propagation. For example, this is how increasing node separations are simulated at Rutgers University's WINLAB ORBIT grid, a testbed with hundreds of closely spaced wireless nodes [9]. The main problem with this approach is that it ignores the delays caused by long distance propagation.

In some cases, even distances of only one or two miles can cause delay problems. For example, when $802.11 \mathrm{~b}$ is operating in the standard access point-client setting (managed mode), the Short Inter-Frame Spacing (SIFS) interval is only $10 \mu \mathrm{s}$. Within this interval, packets must be sent and acknowledged, limiting the maximum distance to less than one mile. For higher performance $802.11 \mathrm{~g}$ and $802.11 \mathrm{a}$, the SIFS interval is $4.5 \mu \mathrm{s}$, limiting the range further [10]. These limitations are at the data link layer level, and so enabling or shortening network layer (e.g. ping) timeouts alone cannot help test long distance issues.

Instead, by physically simulating long distance propagation, these link layer issues can be tested directly. The cheapest way to create very long RF delay lines is to modulate an optical carrier and use fiber optic cable. Even when using higher cost single mode fiber, the cost per foot is lower than that of thick RF cable. An additional benefit is that long fiber runs are essentially lossless, even at several mile lengths". So, propagation delay issues can be separated from path loss and misaligned antenna issues.

Varying fiber optic lengths during testing is somewhat awkward, but a straightforward way is to treat each fiber link like a different attenuator value. Then, with an RF switch on either end, each converter-fiber-converter channel can be switched in and out to affect the transmission length. Unlike directional testing, there is little need for continuous distance variation or even testing

* RF-to-optical conversion typically includes an insertion loss of $20-30 \mathrm{~dB}$, but the extremely long lengths of fiber optics that follow add little attenuation. 
across more than two or three lengths. Instead, only a few converter-fiber-converter channels are needed to test 1) whether the radios work the target ranges and 2) what higher layer effects (e.g. quality of service changes) occur at short versus long distances. The total physical simulation system is shown in the figure below.

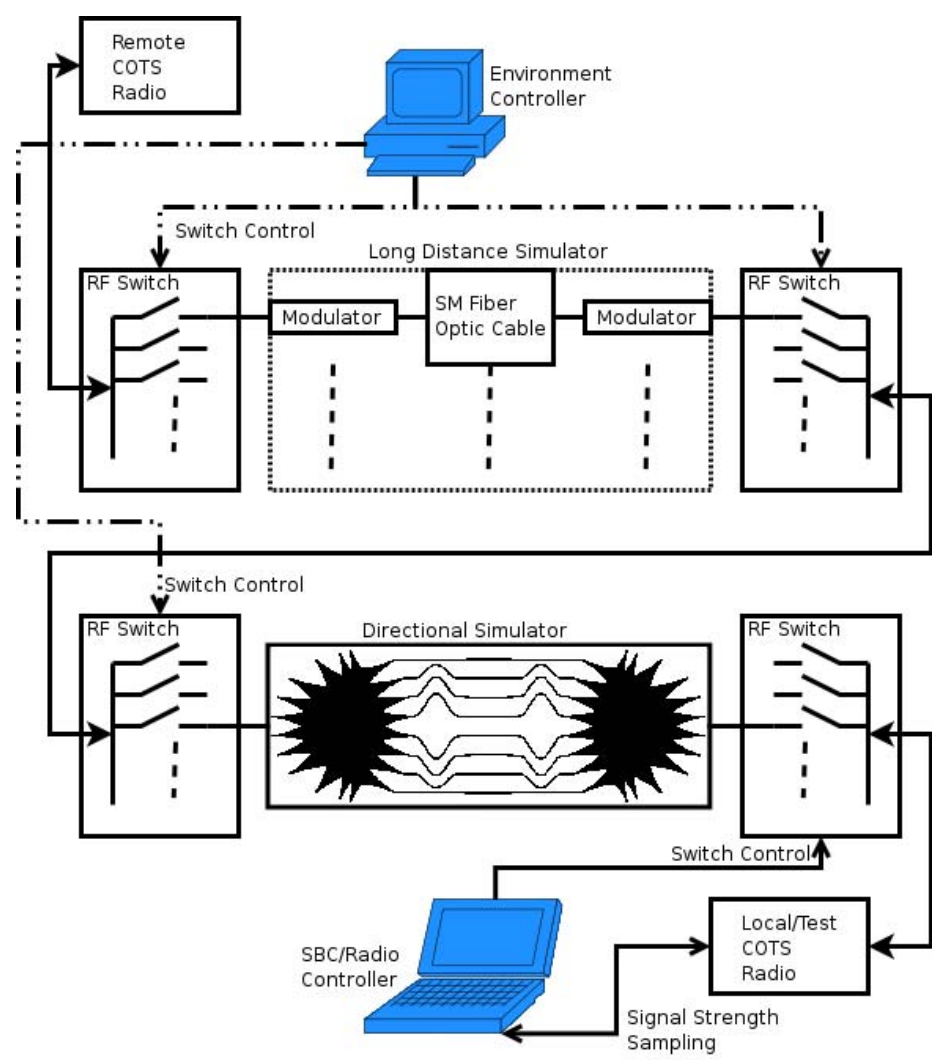

Figure 2: Total Directional and Long Distance Channel Simulator Block Diagram

\section{CURRENT IMPLEMENTATION AND USE}

At SSC-SD, we implemented the directional simulation capability to reduce the need for outdoor testing. The wireless communications system we developed uses a COTS Proxim Tsunami MP.11 radio, which is an 802.11a radio modified for long range and specialized roaming. To that, we added a Rotman lens-based phased array antenna to provide electronically-steerable high gain. As such, the back-to-back RF lens simulator was straightforward. The modulated fiber option for long distance simulation was also available through other groups at SSC Pacific, but at that point we had already determined that the Proxim radios would work at range.

One of our simulation systems is shown in the figure below. The Rotman lenses are in stripline and include nested cosine wires to straighten out the antenna contour for ease of connecting together or to a linear array antenna [11]. RF cables connect the feed ports to connectorized GaAs 1x8 RF switches that are powered and controlled by microcontroller boards (one shown on top of the foreground lens). These boards are powered and controlled via USB by computers: the environment controller on one side and a single board computer (SBC) associated with the Proxim radio on the other. The common RF ports on either side are attached to their respective radios.

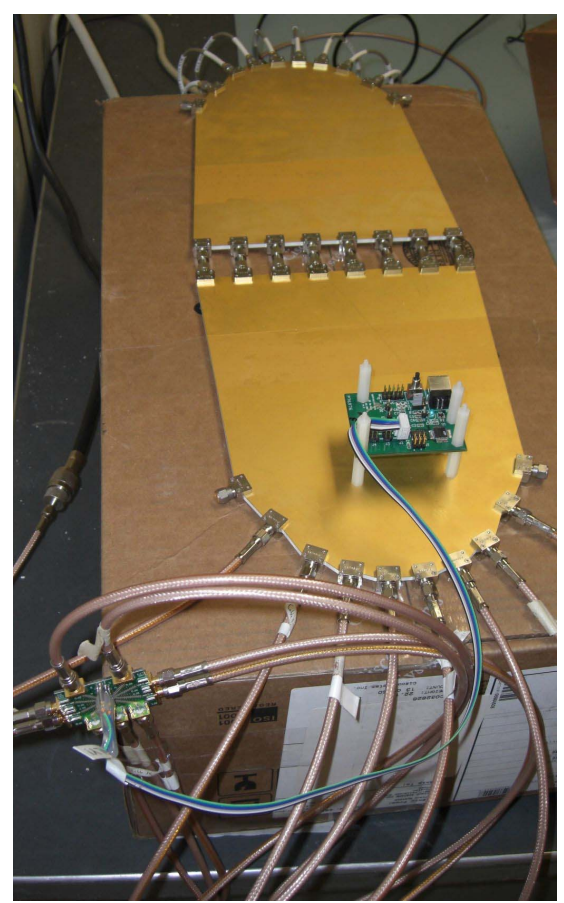

Figure 3: Rotman Lens Implementation of Directional Channel Simulator

This simulator allowed for rapid development and testing of neighbor discovery and tracking software for the system. Having a lab testing capability allowed us to learn important lessons quickly, including:

- Minimum beam sampling time; below this, reported signal strength readings from the radio are unreliable.

- Maximum off-beam sampling time; beyond this, the radio may enter the "slow scan" roaming state, which severely affects latency.

- Initial (neighbor) discovery issues and ways to coordinate optimization once a link is established.

- Tradeoffs between scheduled and reactive beam sampling for the tracking loop.

We also developed a slightly more complicated version that included two sets of back-to-back RF lenses that connected to two variable attenuators and a power divider/combiner on the environment controller side. On 
the radio side, each lens chain connected to a different radio. Each radio and associated $\mathrm{SBC} /$ directional antenna covered a $90^{\circ}$ sector. So, we were able to test not only discovery and tracking on both radios but also sector-tosector handover. All of this testing was invaluable to the rapid prototyping of a wireless system that was demonstrated at the 2008 Trident Warrior naval exercises.

\section{LIMITATIONS AND POSSIBLE IMPROVEMENTS}

A drawback of the currently implemented simulators is that the beam positions on the lenses are fixed. Rolloff between the beams is sharp, around $10 \mathrm{~dB}$, and since the environment controller is limited to these beam positions, the variations are unnecessarily abrupt. Thus, testing under these conditions represents a worst-case scenario: instantaneous rotation of the remote platform by a full beamwidth (e.g. $12^{\circ}$ ). We found that our tracking and handover algorithms worked better outdoors simply due to the more gradual variation in signal strength. As mentioned earlier, a waveguide lens with a mechanically sliding horn or additional phase shifters can help create continuous phase variation on the environment controller side.

Furthermore, for the dual channel implementation, switching two variable attenuators to force handover is awkward and requires a total of four RF lenses. A possible solution is to use two larger back-to-back lenses with twice the number of ports. Then, each radio can be tied to half of the lens ports and handover between sectors remains a spatial steering phenomenon. Though the steering directions may no longer map to real world angles, the simulation should be good enough to develop and test tracking and handover algorithms.

\section{CONCLUSION}

In order to leverage low cost, high performance COTS radio technologies for military use, high gain directional antennas are need to close the link at long range. It is overly expensive and time consuming to model such radios in software or to repeatedly preform full hardware tests in operational or near-operational environments. Instead, by using physical systems to simulate the directional and long distance environments, much can be learned quickly and at reduced cost. Directional antenna systems or multiple radio architectures can be tested using either back-to-back RF lenses or variable attenuators. Long distance timing issues can be tested using RF optical modulators and miles of low cost fiber optics. The SUT is then tested using an environment controller that chooses amongst beam directions and transmission lengths. Such a testbed has been developed by SSC Pacific and was used to successfully develop and test a directional wireless system.

\section{REFERENCES}

[1] C. Cirullo et al., "Network Solutions for Employing COTS Radios in Multi-Sector Directional Architectures," Forthcoming MILCOM 2008. November 2008.

[2] OPNET, [Available Online at] http://www.opnet.com/.

[3] Qualnet, [Available Online at] http://www.scalablenetworks.com/.

[4] Z. Zhang, "DTRA: Directional Transmission and Reception Algorithms in WLANs with Directional Antennas for QoS Support," IEEE Network, May/June 2005, pp. 27-32.

[5] G. Jakllari et al., "An Integrated Neighbor Discovery and MAC Protocol for Ad Hoc Networks Using Directional Antennas," IEEE International Symposium on a WoWMoM 2005, June 2005.

[6] A. Shum et al., "Directional Ad Hoc Networking (DANTE) Project--Literature and Protocol Design Overview," SSC Pacific internal project report, August 2006.

[7] Georgia Institute of Technology Short Course on Phased Array Antennas for Radar and Communications

[8] W. Rotman and R. F. Turner, "Wide-Angle Microwave Lens for Line Source Applications," IEEE Transactions on Antennas and Propagation, vol. 11, issue 6, November 1963.

[9] Discussion with Wade Trappe at the presentation of the paper: D. Rastogi et al., "A Comparative Study of AODV and OLSR on the ORBIT Testbed," MILCOM 2007. October 2007.

[10] H. Kazemi, "Long Range Throughput - Real World Experiences," [Available Online at] http://www.seattlewireless.net/pipermail/dev/2005January/021582.html, Downloaded June 15, 1007.

[11] C. Meagher et al., "Real-World Rotman Lens Prototyping and Analysis," Forthcoming Antennas and Propagation International Symposium, 2008 IEEE, July 2007. 